\title{
miR-96 inhibits cardiac hypertrophy by targeting growth factor receptor-bound 2
}

\author{
Y. Xia' ${ }^{1}$, J. Sheng' ${ }^{2}$, G.Y. Liang ${ }^{1}$, D.X. Liu' ${ }^{1}$, Q. Tang ${ }^{2}$ and A.P. Cheng ${ }^{2}$ \\ 1'Department of Cardio-Thoracic Surgery, Affiliated Hospital of Zunyi Medical College, \\ Zunyi, China \\ ${ }^{2}$ Department of Cardiology, Affiliated Hospital of Zunyi Medical College, Zunyi, China
}

Corresponding author: J. Sheng

E-mail: 570140436@qq.com

Genet. Mol. Res. 14 (4): 18958-18964 (2015)

Received August 8, 2015

Accepted October 26, 2015

Published December 29, 2015

DOI http://dx.doi.org/10.4238/2015.December.29.2

\begin{abstract}
Increasing evidence has indicated that microRNAs are involved in the pathogenesis of cardiac hypertrophy. However, whether miR-96 is involved in heart diseases, particularly cardiac hypertrophy, remains unclear. In this study, we found that miR-96 is a negative regulator of cardiac hypertrophy. In primary cardiomyocytes, overexpression of miR-96 inhibited phenylephrine-induced cardiomyocyte hypertrophy and decreased the mRNA expression of cardiac hypertrophy markers such as atrial natriuretic factor and $\beta$-myosin heavy chain. Interestingly, we found that growth factor receptor-bound 2 is a direct target of miR-96, which is a negative regulator of cardiac hypertrophy. Overexpression of miR-96 in cardiomyocytes led to reduced growth factor receptor-bound 2 expression. More importantly, miR-96 repressed the extracellular-regulated protein kinase signaling pathway by targeting growth factor receptor-bound 2 in cardiomyocytes. Our data demonstrate that miR-96 is a negative regulator of cardiac hypertrophy and extracellular-regulated protein kinase signaling, thus offering a new therapeutic strategy for cardiac hypertrophy.
\end{abstract}

Key words: Cardiac hypertrophy; Growth factor receptor-bound 2; miR-96 


\section{INTRODUCTION}

Cardiomyocyte hypertrophy is a hallmark of many heart diseases (Frey and Olson, 2003; Voulgari et al., 2010). While heart function may benefit from cardiac hypertrophy at an early stage, long-term hypertrophic stimuli can lead to heart failure (Heineke and Molkentin, 2006). Although genetic and environmental factors are considered important, the molecular mechanisms of cardiomyocyte hypertrophy remain unclear. Thus, it is important to understand the molecular mechanism of cardiac hypertrophy and to explore effective intervention targets.

MicroRNAs (miRNAs) are small noncoding RNAs that are 19-22 nucleotides in length and negatively regulate the expression of target genes by binding to the 3'-untranslated region (3'-UTR) of target mRNAs (Bartel, 2004). An increasing number of studies have shown that miRNAs are involved in cardiac hypertrophy. For example, miR-378 is a key regulator of cardiac hypertrophy and represses mitogen-activated protein kinase pathway factor (Ganesan et al., 2013; Nagalingam et al., 2013; Nagalingam et al., 2014). miR-133 can suppress cardiac hypertrophy by binding to its targets RhoA, Cdc42, and Nelf-A/WHSC2 (Care et al., 2007). In contrast, miR-23a is required for cardiomyocyte hypertrophy (Lin et al., 2009). Thus, it appears that miRNAs play pivotal roles in regulating cardiac hypertrophy. However, it remains unclear whether other miRNAs regulate cardiac hypertrophy.

It has previously been reported that miR-96 is involved in many diseases and biological processes. miR-96 was found to be overexpressed in many types of cancer and to promote cancer cell proliferation, metastasis, and metabolism (Guo et al., 2014; Yu et al., 2014; Zhang et al., 2014). In addition, miR-96, together with miR-182 and miR-183, are highly expressed in the retina and are associated with syndromic retinal degeneration (Lumayag et al., 2013). Moreover, a mutation in the seed region of miR-96 can result in progressive hearing loss (Lewis et al., 2009; Mencia et al., 2009). However, whether miR-96 is involved in heart diseases, particularly cardiac hypertrophy, is unknown.

In this study, we examined the function of miR-96 in cardiac hypertrophy. We found that miR-96 repressed cardiac hypertrophy by directly targeting growth factor receptor-bound 2 (GRB2), an important regulator of cardiac hypertrophy. We identified a new role for miR-96 in the process of cardiac hypertrophy and that miR-96 may be a new therapeutic target for heart diseases.

\section{MATERIAL AND METHODS}

\section{Cell culture, transfection, and cell surface area measurement}

Primary neonatal cardiomyocytes were isolated from 1-day-old Sprague-Dawley rats as described previously (Ganesan et al., 2013). Isolated cardiomyocytes and 293T cells (American Type Culture Collection, Manassas, VA, USA) were cultured in Dulbecco's modified Eagle's medium supplemented with $10 \%$ fetal bovine serum, $1 \mathrm{mM}$ glutamine, $100 \mathrm{U} / \mathrm{mL}$ penicillin, and 100 $\mathrm{mg} / \mathrm{mL}$ streptomycin.

Transfection was performed using Lipofectamine 2000 , and $50 \mu \mathrm{M}$ phenylephrine (SigmaAldrich, St. Louis, MO, USA) or an equal volume of phosphate-buffered saline was added $48 \mathrm{~h}$ after transfection. Cells were harvested $48 \mathrm{~h}$ after stimulation.

The cell surface area of $\alpha$-actin-stained cells or unstained cells was measured as described previously (Ganesan et al., 2013). A total of 100-200 cardiomyocytes in 20-50 fields were examined in each experiment. The Image $\mathrm{J}$ software was used to measure the area of each cell (NIH, Bethesda, MD, USA). 


\section{Plasmid, siRNA oligos, and luciferase assay}

To construct luciferase reporter plasmids, the 3'-UTR fragment of GRB2 was amplified from mouse cDNA and inserted into the pRL-TK vector. MicroRNA double-stranded mimics, antagomir, and siRNA oligos were purchased from Genepharma (Suzhou, China).

293T cells were added to a 24-well plate and co-transfected with miR-96 mimics or the negative control, pRL-TK-GRB2-3'-UTR and pGL3-basic reporter plasmids. At $48 \mathrm{~h}$ after transfection, cells were lysed and the relative luciferase activity was analyzed using the Dual Luciferase Reporter Assay System (Promega, Madison, WI, USA) on a luminometer (Promega).

\section{Real-time polymerase chain reaction (PCR)}

RNA was isolated using Trizol reagent from cells, and 500 ng RNA was reverse-transcribed into cDNA using the polyA RT system. Real-Time PCR was performed on an ABI 7500 Fast Real Time PCR system (Applied Biosystems, Foster City, CA, USA). Then, $4 \mu \mathrm{L}$ cDNA, $1 \mu \mathrm{L}$ primer and $5 \mu \mathrm{L}$ Sybr Green (Applied Biosystems, Foster City, CA, USA) were mixed for real-time PCR using the following procedures: Step $1: 95^{\circ} \mathrm{C} 25 \mathrm{~s}$, Step $2: 62^{\circ} \mathrm{C} 30 \mathrm{~s}$, Step $3: 72^{\circ} \mathrm{C} 30 \mathrm{~s}$, run 40 cycles. The following primers were used in this study: atrial natriuretic factor (ANF)-F: GCTTCCAGGCCA TATTGGAG; ANF-R: GGGGGCATGACCTCATCTT; $\beta$-myosin heavy chain (MHC)-F: ACTGTCAA CACTAAGAGGGTCA; $\beta$-MHC-R: TTGGATGATTTGATCTTCCAGGG; glyceraldehyde 3-phosphate dehydrogenase-F: TGGATTTGGACGCATTGGTC; glyceraldehyde 3-phosphate dehydrogenase-R: TTTGCACTGGTACGTGTTGAT; miR-96: TCATATTTGGCGATGGCTT; uni-primer: CGAATTCTAGAGCTCGAGGCAGG.

\section{Western blotting}

Cardiomyocytes were lysed in RIPA lysis buffer containing $10 \mathrm{mM}$ Tris- $\mathrm{HCl}, \mathrm{pH} 7.5,1 \%$ sodium dodecyl sulfate, $1 \mathrm{mM} \mathrm{Na}_{3} \mathrm{VO}_{4}, 10 \mathrm{mM} \mathrm{NaF}$, and protease inhibitor cocktail (Roche, Basel, Switzerland). Next, $20 \mathrm{mg}$ protein samples were separated by sodium dodecyl sulfate-polyacrylamide gel electrophoresis according to standard procedures. Anti-GRB2 (Cell Signaling Technology, Danvers, MA, USA), anti-extracellular regulated protein kinase (ERK) 1/2 (Cell Signaling), anti-p-ERK1/2 (Cell Signaling), anti- $\alpha$-actin (Sigma-Aldrich), and anti- $\alpha$-tubulin (Sigma-Aldrich) were used for western blotting.

\section{Statistical analysis}

All results are reported as means \pm SE from at least 3 independent experiments. The Student $t$-test was used to analyze the differences in mean values between 2 groups using GraphPad Prism 5.0 (GraphPad Software, Inc., La Jolla, CA, USA). Statistical significance was considered at $P<0.05$.

\section{RESULTS}

miR-96 inhibited hypertrophy of cardiomyocytes

Phenylephrine can induce hypertrophy of cardiomyocytes (Ganesan et al., 2013), and 
thus we attempted to determine whether miR-96 participate in hypertrophic signaling pathways. As shown in Figure 1A, we found that miR inhibited hypertrophy of cardiomyocytes. However, phenylephrine-induced cardiomyocyte hypertrophy was also suppressed after transfection with miR-96 mimics. Because ANF and $\beta-\mathrm{MHC}$ are known to be hallmarks of cardiomyocyte hypertrophy, the mRNA expression of ANF and $\beta-M H C$ was determined by performing reverse transcription-PCR. We observed that the mRNA levels of ANF and $\beta-M H C$ were repressed in cardiomyocytes transfected with miR-96 mimics (Figure 1B). These results demonstrated that overexpression of miR-96 inhibited the hypertrophy of cardiomyocytes.
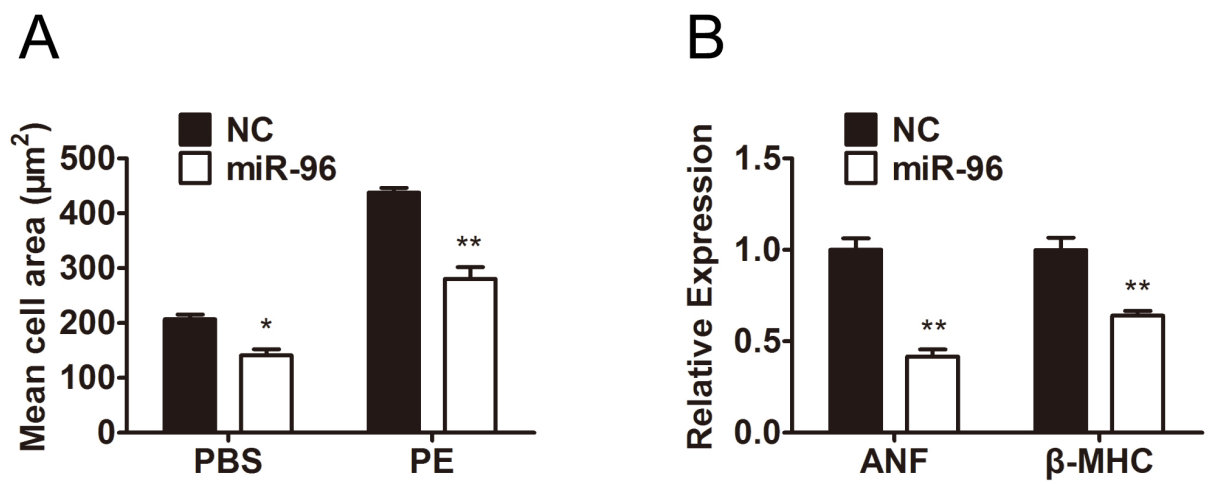

Figure 1. miR-96 inhibits cardiomyocyte hypertrophy. A. Cardiomyocytes were transfected with miR-96 mimics or negative control oligos (NC), and $48 \mathrm{~h}$ later, $50 \mu \mathrm{M}$ phenylephrine (PE) or equal volume of phosphate-buffered saline was added, and then cell surface area was analyzed. B. Reverse transcription-PCR analysis of ANF and $\beta-M H C$ mRNA levels in cardiomyocytes transfected with miR-96 mimics or negative control oligos (NC). Data are reported as means \pm SE of triplicate measurements. ${ }^{*} \mathrm{P}<0.05,{ }^{* *} \mathrm{P}<0.01$.

\section{GRB2 was directly targeted by miR-96 in cardiomyocytes}

Based on the previous studies, we hypothesized that miR-96 may target key regulators in cardiomyocyte hypertrophy. Interestingly, using TargetScan, we found that GRB2, which has been reported to be a key regulator of cardiomyocyte hypertrophy, is a potential target of miR-96 acrossspecies (Figure 2A). To determine whether GRB2 is a direct target of miR-96, we analyzed the mRNA and protein levels of GRB2 in cardiomyocytes transfected with miR-96 mimics. The mRNA and protein levels of GRB2 were effectively repressed by miR-96 (Figure 2B and C). We also evaluated the luciferase activity of a reporter plasmid carrying the 3'-UTR of GRB2. As shown in Figure 2D, miR-96 significantly reduced luciferase activity compared with negative control oligos. Our data showed that miR-96 directly targeted GRB2 in cardiomyocytes.

\section{miR-96 repressed the ERK signaling pathway by targeting GRB2}

The positive regulation of GRB2 in cardiomyocyte hypertrophy has been suggested in previous studies (Zhang et al., 2003), and thus we evaluated the effect of si-GRB2 on cardiomyocyte hypertrophy. We found that si-GRB2 could repress phenylephrine-induced cardiomyocyte hypertrophy, which is similar to the effect observed following miR-96 mimic transfection. In addition, we found that si-GRB2 could rescue the effect of miR-96 antagomir on the cardiomyocyte surface area (Figure 3A). GRB2 is an upstream regulator of ERK, which also plays a pivotal role in cardiomyo- 
cyte hypertrophy. Thus, we hypothesized that miR-96 could inhibit p-ERK expression by targeting GRB2 in cardiomyocytes. To test this hypothesis, we investigated the effects of miR-96 mimics and miR-96 antagomir on the expression of p-ERK and GRB2. As shown in Figure 3B, we found that overexpression of miR-96 decreased the expression of p-ERK and GBR2. Additionally, knockdown of miR-96 led to increased p-ERK and GBR2 expression (Figure 3B). These results suggested that miR-96 repressed ERK signaling by targeting GRB2 in cardiomyocytes.

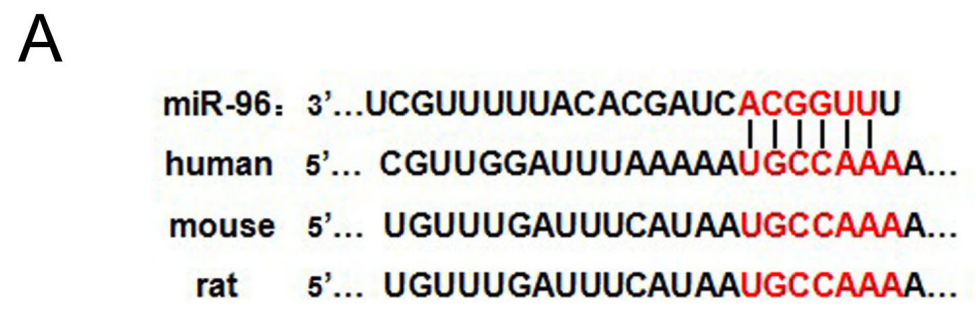

B
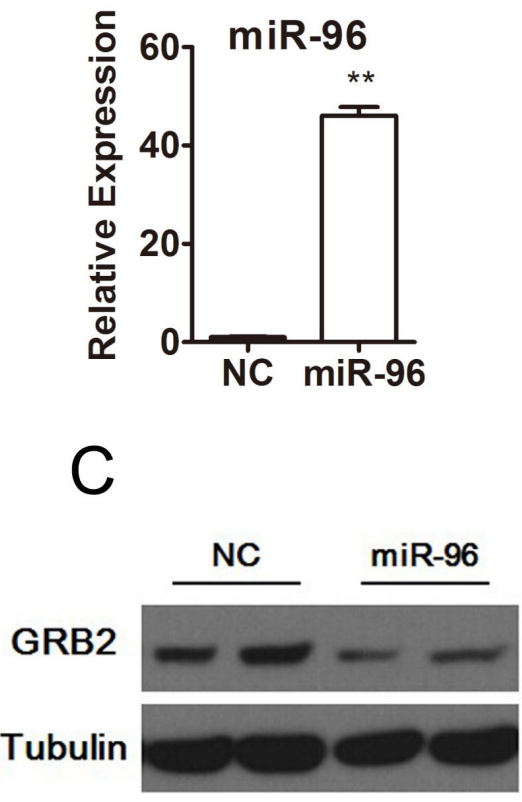
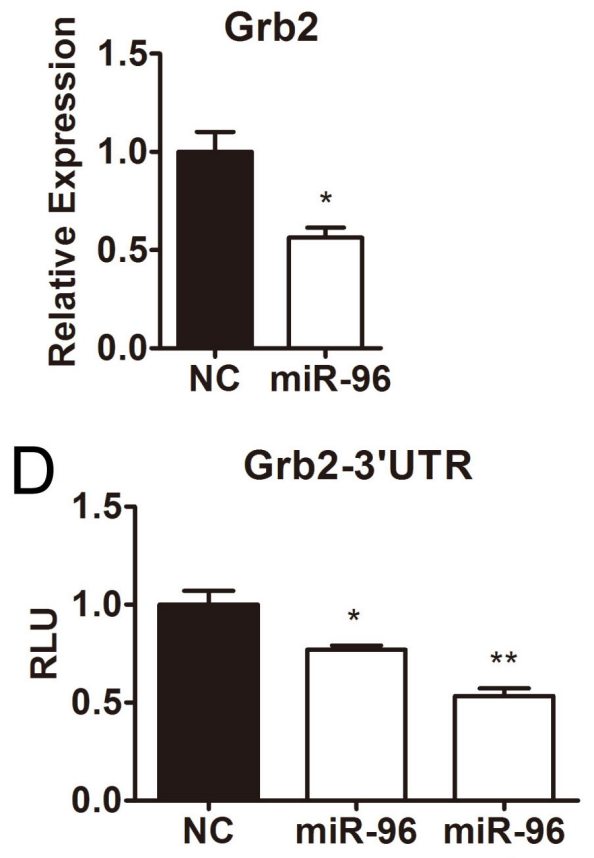

Figure 2. GRB2 is a direct target of miR-96 in cardiomyocytes. A. Sequences of predicted miR-96 binding sites in GRB2 3'-UTR across species. B. Reverse transcription-PCR analysis of miR-96 and GRB2 mRNA levels in cardiomyocytes transfected with miR-96 mimics or negative control oligos (NC). C. Western blot analysis of GRB2 expression in cardiomyocytes miR-96 mimics or negative control oligos (NC); $\alpha$-tubulin was used as a loading control. D. Luciferase activity in cardiomyocytes co-transfected with different amounts of miR-96 mimics or negative control oligos (NC), pRLTK-GRB2-3'-UTR and pGL3-basic. pGL3-basic was used for transfection normalization. Data are reported as means \pm SE of triplicate measurements. ${ }^{*} P<0.05,{ }^{*} \mathrm{P}<0.01$. 

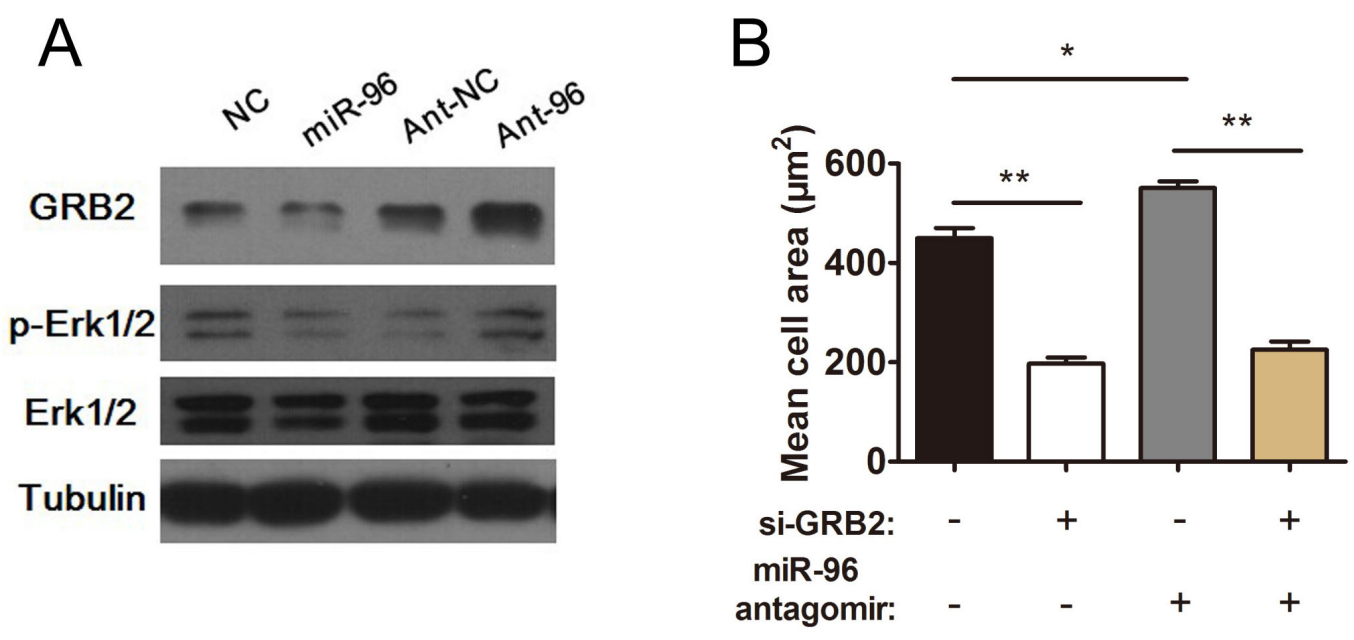

Figure 3. miR-96 inhibits ERK signaling pathway by targeting GRB2. A. Cardiomyocytes were transfected with siGRB2 oligos or si-NC control oligos and miR-96 antagomir or antagomir negative control (a-NC), and $48 \mathrm{~h}$ later, 50 $\mu \mathrm{M}$ phenylephrine (PE) was added, and then cell surface area was analyzed. B. Western blot analysis of GRB2, p-ERK1/2, and ERK1/2 expression in cardiomyocytes transfected with miR-96 mimics, negative control oligos (NC), miR-96 antagomir (Ant-96), and antagomir negative control (Ant-NC); $\alpha$-tubulin was used as a loading control. Data are reported as means \pm SE of triplicate measurements. ${ }^{*} \mathrm{P}<0.05$, ${ }^{* *} \mathrm{P}<0.01$.

\section{DISCUSSION}

miRNAs have been found to be important posttranscriptional regulators, which participate in many basic cell functions, including cardiac hypertrophy. In this study, we found that miR-96 negatively regulated the development of cardiac hypertrophy. Overexpression of miR-96 in primary cardiomyocytes inhibited phenylephrine-induced hypertrophy and decreased the mRNA expression of cardiac hypertrophy markers such as ANF and $\beta-\mathrm{MHC}$. This is the first study to demonstrate the function of miR-96 in cardiomyocytes.

Additionally, we found miR-96 directly targeted GRB2. GRB2 plays a positive role in receptor tyrosine kinase signaling. GRB2 is the key link between epidermal growth factor receptor and the Ras-mitogen-activated protein kinase signaling pathway (Buday and Downward, 1993; Gale et al., 1993; Lin et al., 2012). A previous study showed that the GRB2-p38 MAPK pathway is essential for cardiac hypertrophy in response to pressure overload (Zhang et al., 2003). Similarly, miR-378 was found to inhibit cardiac hypertrophy by targeting GRB2.

ERK signaling is known to play a significant role in pathology. GRB2 is an upstream regulator of ERK signaling. Our results suggest that miR-96 directly targets GRB2 and represses GRB2induced activation of ERK. Based on these findings, miR-96 may achieve its physiologicalfunction by inhibiting the GRB2-ERK signaling pathway in cardiomyocytes. This explanation is consistent with the results of a previous study, which showed that ERK promotes cardiac hypertrophy in a transgenic mouse model (Bueno et al., 2000).

In summary, our results provide the first evidence that miR-96 may block cardiac hypertrophy by targeting GRB2. However, the in vivo effect of miR-96 on cardiomyopathy and whether miR-96 has other targets involved in cardiac hypertrophy remain unknown. Our findings provide a novel strategy for the treatment of cardiac hypertrophy. 


\section{Conflicts of interest}

The authors declare no conflict of interest.

\section{REFERENCES}

Bartel DP (2004). MicroRNAs: genomics, biogenesis, mechanism, and function. Cell 116: 281-297.

Buday L and Downward J (1993). Epidermal growth factor regulates p21ras through the formation of a complex of receptor, Grb2 adapter protein, and Sos nucleotide exchange factor. Cell 73: 611-620.

Bueno OF, De Windt LJ, Tymitz KM, Witt SA, et al. (2000). The MEK1-ERK1/2 signaling pathway promotes compensated cardiac hypertrophy in transgenic mice. EMBO J. 19: 6341-6350.

Carè A, Catalucci D, Felicetti F, Bonci D, et al. (2007). MicroRNA-133 controls cardiac hypertrophy. Nat. Med.13: 613-618.

Frey N and Olson EN (2003). Cardiac hypertrophy: the good, the bad, and the ugly. Annu. Rev. Physiol. 65: 45-79.

Gale NW, Kaplan S, Lowenstein EJ, Schlessinger J, et al. (1993). Grb2 mediates the EGF-dependent activation of guanine nucleotide exchange on Ras. Nature 363: 88-92.

Ganesan J, Ramanujam D, Sassi Y, Ahles A, et al. (2013). MiR-378 controls cardiac hypertrophy by combined repression of mitogen-activated protein kinase pathway factors. Circulation 127: 2097-2106.

Guo H, Li Q, Li W, Zheng T, et al. (2014). MiR-96 downregulates RECK to promote growth and motility of non-small cell lung cancer cells. Mol. Cell. Biochem. 390: 155-160.

Heineke J and Molkentin JD (2006). Regulation of cardiac hypertrophy by intracellular signalling pathways. Nat. Rev. Mol. Cell. Biol. 7: 589-600.

Lewis MA, Quint E, Glazier AM, Fuchs H, et al. (2009). An ENU-induced mutation of miR-96 associated with progressive hearing loss in mice. Nat. Genet. 41: 614-618.

Lin CC, Melo FA, Ghosh R, Suen KM, et al. (2012). Inhibition of basal FGF receptor signaling by dimeric Grb2. Cell 149: 1514-1524.

Lin Z, Murtaza I, Wang K, Jiao J, et al. (2009). miR-23a functions downstream of NFATc3 to regulate cardiac hypertrophy. Proc. Natl. Acad. Sci. U. S. A. 106: 12103-12108.

Lumayag S, Haldin CE, Corbett NJ, Wahlin KJ, et al. (2013). Inactivation of the microRNA-183/96/182 cluster results in syndromic retinal degeneration. Proc. Natl. Acad. Sci. U. S. A. 110: E507-E516.

Mencía A, Modamio-Høybjor S, Redshaw N, Morín M, et al. (2009). Mutations in the seed region of human miR-96 are responsible for nonsyndromic progressive hearing loss. Nat. Genet. 41: 609-613.

Nagalingam RS, Sundaresan NR, Gupta MP, Geenen DL, et al. (2013). A cardiac-enriched microRNA, miR-378, blocks cardiac hypertrophy by targeting Ras signaling. J. Biol. Chem. 288: 11216-11232.

Nagalingam RS, Sundaresan NR, Noor M, Gupta MP, et al. (2014). Deficiency of cardiomyocyte-specific microRNA-378 contributes to the development of cardiac fibrosis involving a transforming growth factor beta (TGFb1)-dependent paracrine mechanism. J. Biol. Chem. 289: 27199-27214.

Voulgari C, Papadogiannis D and Tentolouris N (2010). Diabetic cardiomyopathy: from the pathophysiology of the cardiac myocytes to current diagnosis and management strategies. Vasc. Health Risk Manag. 6: 883-903.

Yu JJ, Wu YX, Zhao FJ and Xia SJ (2014). miR-96 promotes cell proliferation and clonogenicity by down-regulating of FOXO1 in prostate cancer cells. Med. Oncol. 31: 910.

Zhang J, Kong X, Li J, Luo Q, et al. (2014). miR-96 promotes tumor proliferation and invasion by targeting RECK in breast cancer. Oncol. Rep. 31: 1357-1363.

Zhang S, Weinheimer C, Courtois M, Kovacs A, et al. (2003). The role of the Grb2-p38 MAPK signaling pathway in cardiac hypertrophy and fibrosis. J. Clin. Invest. 111: 833-841. 